\title{
Insufficient Mitigation of Bone Loss by Zoledronic Acid after Treatment with Denosumab
}

\author{
Jung-Wee Park \\ Department of Orthopaedic Surgery, Seoul National University Bundang Hospital, Seoul National University College of Medicine, \\ Seongnam, Korea
}

Corresponding author

Jung-Wee Park

Department of Orthopaedic Surgery, Seoul

National University Bundang Hospital, Seoul

National University College of Medicine,

82 Gumi-ro 173 beon-gil, Bundang-gu,

Seongnam 13620, Korea

Tel: +82-31-787-7204

Fax: +82-31-787-4056

E-mail: jwepark@gmail.com

Received: November 2, 2021

Accepted: November 10, 2021
Copyright $@ 2021$ The Korean Society for Bone and Mineral Research

This is an Open Access article distributed under the terms of the Creative Commons Attribution Non-Commercial License (https://creativecommons.org/licenses/by-nc/4.0/) which permits unrestricted non-commercial use, distribution, and reproduction in any medium, provided the original work is properly cited.

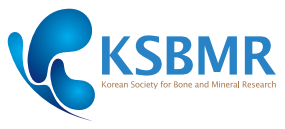

\section{TOTHE EDITOR:}

I read with great interest the article by Kadaru and Shibli-Rahhal [1] on the bone loss related to zoledronic acid (ZA) after denosumab treatment. They reported bone mineral density (BMD) changes in 12 patients who were treated with ZA after denosumab and found a significant decline in BMD at femoral neck. The authors have delivered a clinically important and interesting message.

Medical treatment for osteoporosis after cessation of denosumab is becoming more crucial. Modi et al. [2] reported that $48.8 \%$ and $64.3 \%$ out of 617 patients who were treated with denosumab discontinued treatment at 12 and 24 months, respectively. Other than in patients who have reached T-score of BMD over -2.5 by the denosumab treatment, the discontinuation might be either because of the difficulty in persistent treatment of osteoporosis which is asymptomatic before the osteoporotic fracture or because of the increased risk of atypical femoral fracture (AFF) or medication-related osteonecrosis of the jaw (MRONJ). The rebound effect after denosumab cessation involves the increase of the bone turnover markers (BTMs) and decrease of BMD.[3-6] One of the efforts to avoid the rebound effect was to start bisphosphonate treatment following denosumab discontinuation. Implementing bisphosphonate mitigated BMD loss in several observational studies, $[3,7,8]$ one of which emphasized the beneficial effect of delayed administration of ZA.[7] The present study showed a decline in BMD after the switch of denosumab to $Z A$, and its independence to number of denosumab doses and the interval between the denosumab and ZA. Moreover, the described pathomechanism of incompetence of bisphosphonate after denosumab treatment due to absence of "open bone surfaces" seems plausible.

As stated in the limitations, the small number of included 12 patients is the biggest weakness of this study. The alleviation effect of ZA following denosumab might be underestimated in this study because of the small number of participants. Furthermore, there was no control group in this study where the patients did not receive any antiosteoporotic treatment after denosumab cessation. Leder et al. [9] found maintenance of BMD $\left(-0.6 \mathrm{~g} / \mathrm{cm}^{2}\right.$ in femoral neck, $-0.8 \mathrm{~g} / \mathrm{cm}^{2}$ in total hip, and $-1.2 \mathrm{~g} / \mathrm{cm}^{2}$ in spine) in 28 patients who were treated after the Denosumab and Teriparatide Administration (DATA) study compared to a significant loss of BMD in 
those who were not treated $\left(-4.2 \mathrm{~g} / \mathrm{cm}^{2}\right.$ in femoral neck, $-4.5 \mathrm{~g} / \mathrm{cm}^{2}$ in total hip and $-10.0 \mathrm{~g} / \mathrm{cm}^{2}$ in spine). Interestingly, the authors suggest the loss in BMD is concentrated within 1 year of ZA and it may reach a plateau after a second dose. The number of patients who received second doses of ZA was only 5. It would have been difficult because of the small number of total included patients, but comparing the BMD changes between patients who had osteoporotic fractures and the others could give additional information. Even if the BMD or BTM levels aggravate after changing denosumab to $Z A$, if the risk of osteoporotic fracture does not increase, it may not be a clinically significant issue.

The absence of information on BTMs, serum vitamin D, and calcium levels at each time point is another limitation. The major finding of decrease of BMD when switching denosumab to ZA could be influenced by above factors. As implementation of potent antiresorptive treatment is mandated after denosumab cessation to avoid the rebound effect, evaluating the antiresorptive effect of following treatment is crucial. Previous studies found the decrease of BTMs by $26.2 \%$ (osteocalcin) to $35.2 \%$ (bone-specific alkaline phosphatase) with raloxifene,[10] and decrease of serum C-terminal telopeptide of type I collagen by $53.4 \%$ to $59.9 \%$ with ibandronate.[11] The suggested pathomechanism in the discussion might be also supported by such data. Despite their rarity, the potential risks of AFF and MRONJ are present with both bisphosphonate and denosumab.[12] Nonetheless, there was no information on the complications of using both medications. Comparison with the patients who were treated with anabolic agents such as parathyroid hormones or romosozumab after denosumab therapy could also be informative.

The paper clearly add to the significance of rebound effect of discontinuing denosumab. The authors also provide meaningful outcomes where ZA mitigates the loss of BMD but not sufficiently. For more accurate analysis, however, larger number of study patients and inclusion of laboratory data are needed to support this finding and the assumption that the main loss of BMD occurs within 1 year of $Z A$ injection. Whether the decrease of BMD after shifting denosumab to ZA increases the risk of osteoporotic fractures is not addressed in the article but may be clinically significant.

\section{DECLARATIONS}

\section{Ethics approval and consent to participate Not applicable.}

\section{Conflict of interest}

No potential conflict of interest relevant to this article was reported.

\section{ORCID}

Jung-Wee Park https://orcid.org/0000-0002-4515-1895

\section{REFERENCES}

1. Kadaru T, Shibli-Rahhal A. Zoledronic acid after treatment with denosumab is associated with bone loss within 1 year. J Bone Metab 2021;28:51-8. https://doi.org/10.11005/jbm. 2021.28.1.51.

2. Modi A, Sajjan S, Insinga R, et al. Frequency of discontinuation of injectable osteoporosis therapies in US patients over 2 years. Osteoporos Int 2017;28:1355-63. https://doi. org/10.1007/s00198-016-3886-y.

3. Bone HG, Bolognese MA, Yuen CK, et al. Effects of denosumab treatment and discontinuation on bone mineral density and bone turnover markers in postmenopausal women with low bone mass. J Clin Endocrinol Metab 2011; 96:972-80. https://doi.org/10.1210/jc.2010-1502.

4. McClung MR, Wagman RB, Miller PD, et al. Observations following discontinuation of long-term denosumab therapy. Osteoporos Int 2017;28:1723-32. https://doi.org/10.1007/ s00198-017-3919-1.

5. Miller PD, Bolognese MA, Lewiecki EM, et al. Effect of denosumab on bone density and turnover in postmenopausal women with low bone mass after long-term continued, discontinued, and restarting of therapy: a randomized blinded phase 2 clinical trial. Bone 2008;43:222-9. https://doi. org/10.1016/j.bone.2008.04.007.

6. Popp AW, Varathan $N$, Buffat $H$, et al. Bone mineral density changes after 1 year of denosumab discontinuation in postmenopausal women with long-term denosumab treatment for osteoporosis. Calcif Tissue Int 2018;103:50-4. https:// doi.org/10.1007/s00223-018-0394-4.

7. Horne AM, Mihov B, Reid IR. Bone loss after romosozumab/denosumab: Effects of bisphosphonates. Calcif Tissue Int 2018;103:55-61. https://doi.org/10.1007/s00223-018- 
0404-6.

8. Lehmann T, Aeberli D. Possible protective effect of switching from denosumab to zoledronic acid on vertebral fractures. Osteoporos Int 2017;28:3067-8. https://doi.org/10. 1007/s00198-017-4108-y.

9. Leder BZ, Tsai JN, Jiang LA, et al. Importance of prompt antiresorptive therapy in postmenopausal women discontinuing teriparatide or denosumab: The Denosumab and Teriparatide Follow-up study (DATA-Follow-up). Bone 2017; 98:54-8. https://doi.org/10.1016/j.bone.2017.03.006.

10. Delmas PD, Ensrud KE, Adachi JD, et al. Efficacy of raloxifene on vertebral fracture risk reduction in postmenopaus- al women with osteoporosis: four-year results from a randomized clinical trial. J Clin Endocrinol Metab 2002;87:360917. https://doi.org/10.1210/jcem.87.8.8750.

11. Eisman JA, Civitelli R, Adami S, et al. Efficacy and tolerability of intravenous ibandronate injections in postmenopausal osteoporosis: 2-year results from the DIVA study. J Rheumatol 2008;35:488-97.

12. Nicolatou-Galitis O, Schiødt M, Mendes RA, et al. Medication-related osteonecrosis of the jaw: definition and best practice for prevention, diagnosis, and treatment. Oral Surg Oral Med Oral Pathol Oral Radiol 2019;127:117-35. https:// doi.org/10.1016/j.0000.2018.09.008. 
